\title{
Channel Estimation Techniques in MIMO-OFDM Systems - Review Article
}

\author{
Raspinderjit Kaur Kahlon ${ }^{1}$, Gurpreet Singh Walia ${ }^{2}$, Anu Sheetal ${ }^{3}$ \\ Student, Dept. of Electronics and Communication, Guru Nanak Dev University, \\ Regional Campus Gurdaspur, India ${ }^{1}$ \\ Assistant Professor, Dept. of Electronics and Communication, Guru Nanak Dev University, \\ Regional Campus Gurdaspur, India ${ }^{2}$ \\ Head of Department, Dept. of Electronics and Communication, Guru Nanak Dev University, \\ Regional Campus Gurdaspur, India ${ }^{3}$
}

\begin{abstract}
Recent mobile telecommunication systems are using MIMO collective with OFDM, which is well-known as MIMO-OFDM, to offer robustness and higher spectrum efficiency. The most important challenge in this scenario is to achieve an accurate channel estimation to identify the information symbols, once the receiver must have the channel state information to balance and process the received signal. The main objective of this paper is to study various techniques and analyze for channel estimation in MIMO-OFDM systems.
\end{abstract}

Keywords: MIMO-OFDM, channel estimation, LS, MMSE, interpolation, LS-Spline

\section{INTRODUCTION}

Orthogonal frequency division multiplexing had attained a lot of importance due to its high data rate transmitting In general, the MIMO-OFDM transmitter has $N_{T}$ parallel ability, robustness against frequency-selective fading transmission paths which are very similar to the single channels and simple implementation. Combination of antenna OFDM system, each branch performing serial-toOFDM with multiple antennas has been providing a parallel conversion, pilot insertion, $N$-point IDFT, cyclic significant increase in capacity through the use of extension and signals are up-converted to RF and transmitter and receiver diversity known as MIMO- transmitted. Channel encoder and the digital modulation OFDM. But such system depends on the prior knowledge can also be done per branch. Before transmitting from of channel state information (CSI) at the receiver. MIMO multiple antennas modulated signals are space-time coded systems use multiple antennas to transmit and receive using the Alamouti algorithm [1].

signals. Channel Estimation means to estimate channel At the receiver end, the CP is removed and $N$-point DFT is parameters from the received signal. Pilot symbols that are performed for each receiver branch. Then using Spaceknown to receiver are being used to estimate the channel Time decoding transmitted symbol from each transmitted parameters. The channel has been estimated separately for is combined. To obtain the output signal demodulation and each packet for packet transmission. Pilot symbols are decoding operations are performed.

needed to be inserted into every data packet.

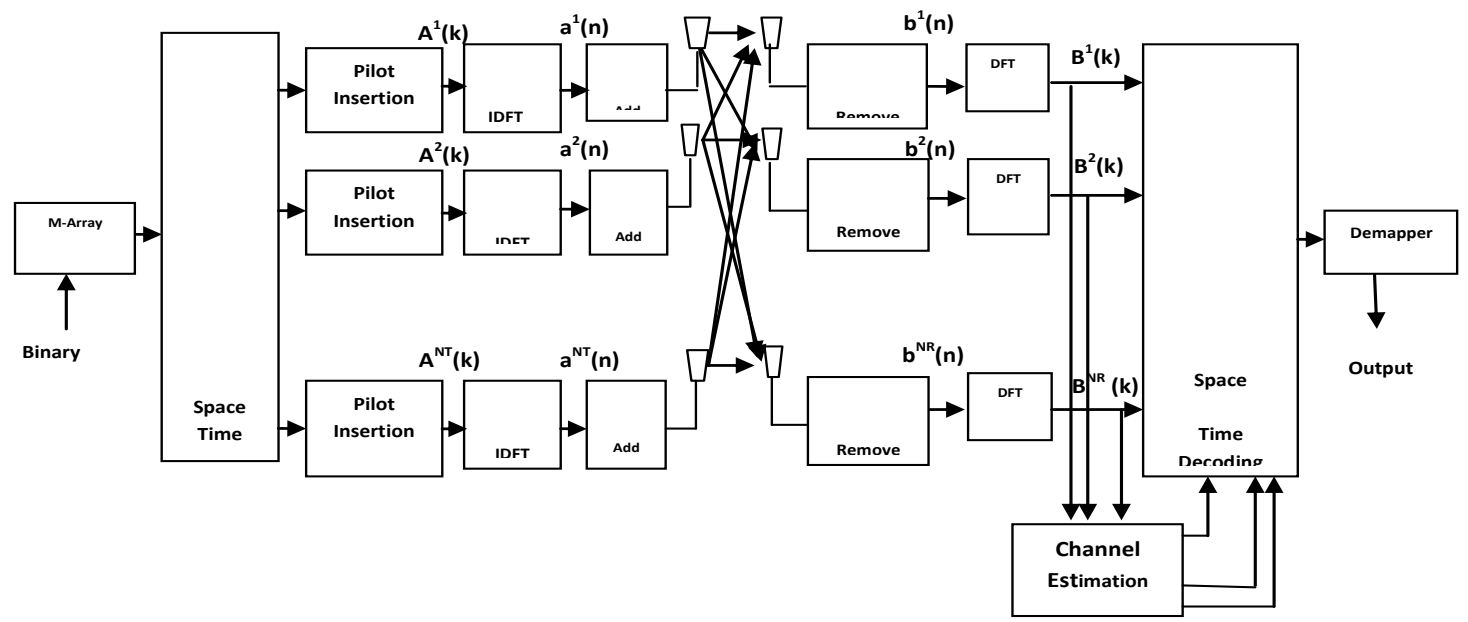

Fig.1 MIMO OFDM System Block Diagram [2] 
For elimination of ISI the guard time interval should be more than the expected largest delay spread of a multipath channel. The transmitted signal will be the convolved with the channel to get the received signal. Assuming that the channel is static during an OFDM block, at the receiver side after removing the $\mathrm{CP}$, the FFT output as the demodulated received signal.

\section{III.CHANNEL ESTIMATION AND TECHNIQUES CHANNEL ESTIMATION}

In an OFDM system, the transmitter modulates the message bit sequence into PSK/QAM symbols, performs IFFT on the symbols to convert them into time-domain signals, and sends them out through a (wireless) channel. The received signal is usually distorted by the channel characteristics. In order to recover the transmitted bits, the channel effect must be estimated and compensated in the receiver. Each subcarrier can be regarded as an independent channel, as long as no ICI (Inter-Carrier Interference) occurs, and thus preserving the orthogonality among subcarriers. The orthogonality allows each subcarrier component of the received signal to be expressed as the product of the transmitted signal and channel frequency response at the subcarrier. Thus, the transmitted signal can be recovered by estimating the channel response just at each subcarrier. In general, the channel can be estimated by using a preamble or pilot symbols known to both transmitter and receiver, which employ various interpolation techniques to estimate the channel response of the subcarriers between pilot tones. In general, data signal as well as training signal, or both, can be used for channel estimation.

\section{A. Pilot Structure}

Depending on the arrangement of pilots, three different types of pilot structures are considered: block type, comb type, and lattice type.

\section{i. Block Type}

A block type of pilot arrangement is depicted in Fig.2. In this type, OFDM symbols with pilots at all subcarriers (referred to as pilot symbols herein) are transmitted periodically for channel estimation. Using these pilots, a time-domain interpolation is performed to estimate the channel along the time axis. As the coherence time is given in an inverse form of the Doppler frequency $f_{\text {Doppler }}$ in the channel, the pilot symbol period must satisfy the following inequality:

$S_{t} \leq \frac{1}{f_{\text {Doppler }}}$

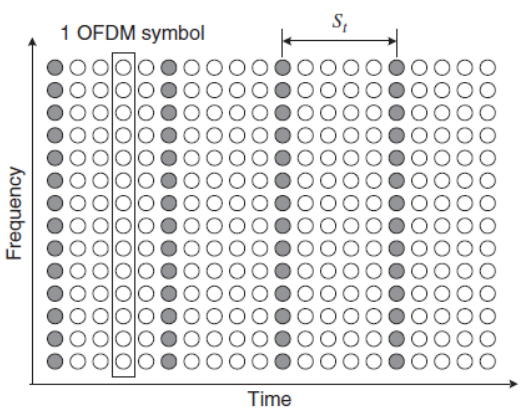

Fig.2 Block type Pilot Arrangement

\section{i. Comb Type}

Comb-type pilot arrangement is depicted in Fig.3. In this type, every OFDM symbol has pilot tones at the periodically-located subcarriers, which are used for a frequency-domain interpolation to estimate the channel along the frequency axis. As the coherence bandwidth is determined by an inverse of the maximum delay spread $\mathrm{S}_{\max }$, the pilot symbol period must satisfy the following inequality:

$$
S_{f} \leq \frac{1}{\sigma_{\max }}
$$

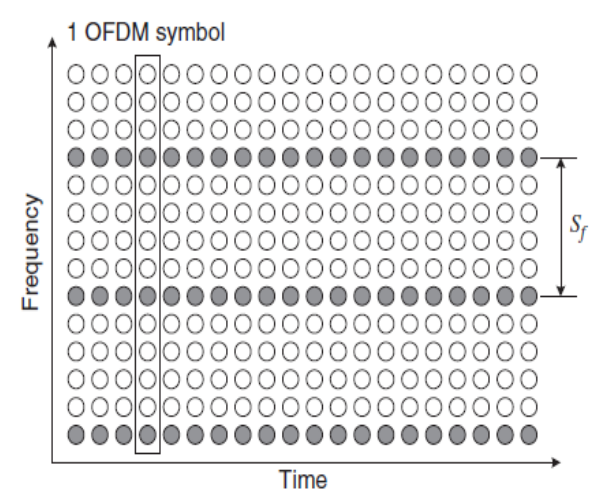

Fig.3 Comb type Pilot Arrangement

\section{B. Training Symbol-Based Channel Estimation}

Training symbols can be used for channel estimation, usually providing a good performance.However, their transmission efficiencies are reduced due to the required overhead of training symbols such as preamble or pilot tones that are transmitted in addition to data symbols.

The least-square (LS) and minimum-mean-square-error (MMSE) techniques are widely used for channel estimation when training symbols are available.

\section{DFT-Based Channel Estimation}

The DFT-based channel estimation technique has been derived to improve the performance of LS or MMSE channel estimation by eliminating the effect of noise outside the maximum channel delay.

Note that the maximum channel delay $L$ must be known in advance. This technique is used for noise reduction.

\section{Decision-Directed Channel Estimation}

Once initial channel estimation is made with the preamble or pilots, the coefficients of channel can be updated with decision-directed (DD) channel estimation, which does not use the preamble or pilots.

DD technique uses the detected signal feedback to track the possibly time-varying channel while subsequently using the channel estimate to detect the signal. 
E. Advanced Channel Estimation Techniques Consider a superimposed signal which adds a training

\section{Channel Estimation Using Superimposed Signal}

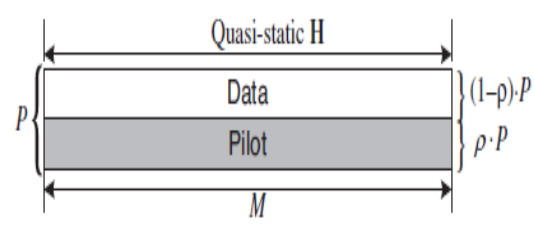

Fig. 7 A superimposed signal for channel estimation.

ii. Channel Estimation in Fast Time-Varying Channels

The channel estimation methods discussed so far may be applicable only when the channel characteristic does not change within an OFDM symbol period.

However, the channel for the terminals that move fast may vary with time within an OFDM symbol period, in which longer OFDM symbol period has a more severe effect on the channel estimation performance.

The time-varying channel may destroy the orthogonality among subcarriers at the receiver, resulting in ICI (InterChannel Interference).

Due to the effect of ICI, it cannot be compensated by the conventional one-tap equalizer. This section deals with the effect of ICI in the time varying channels.

In order to compensate for the effect of ICI under the fastfading channels, we need an accurate estimate of the channel frequency response $\mathrm{H}$.

Although extensive researches have been performed on symbols to extract statistical properties. Furthermore, their estimation of the time-varying channels, most of the performance is usually worse than that of other proposed techniques have been derived and verified under conventional channel estimation techniques that employ a limited condition.

Further research is expected for data transmission in very fast-fading channel environments.

iii. EM Algorithm-Based Channel Estimation The EM (Expectation-Maximization) algorithm has been However, Buss gang algorithm is rarely used in OFDM widely used in a large number of areas that deal with systems since it is not easy to find a nonlinear estimator unknown factors affecting the outcome, such as signal appropriate for the received signal in the OFDM system.

processing, genetics, econometric, clinical, and sociological studies. The EM-based channel estimation is an iterative technique estimators that utilize not only that part of signal for finding maximum likelihood (ML) estimates of a corresponding to the training symbols but also the part channel.

It is classified as a semi-blind method since it can be implemented when transmit symbols are not available.

\section{G. Semi-blind Channel Estimator}

Semi-blind channel estimators are another class of channel

The EM algorithm is particularly useful for channel estimation when available data are incomplete. Incomplete data may be problematic in the situations where insufficient. In MIMO OFDM systems, for

The EM algorithm can convert a multiple-input channel estimation problems. Also, the EM algorithm can be useful interference. The performance of channel estimation at the ell boundary can be improved with additional received time-invariant over D symbol periods.

\section{F. Blind Channel Estimation} or pilot signals. Obviously, such a blind channel estimation technique has an advantage of not incurring an overhead the training signal. It consists of a filter, zero-memory nonlinear estimator, and adaptive algorithm.

Depending on how the zero-memory nonlinear estimator is constructed, it can be classified as Sato algorithm, CMA (Constant Modulus Algorithm), or Godard algorithm. systems since it is not easy to find a nonlinear estimat
appropriate for the received signal in the OFDM system. corresponding to data symbols. In particular, a semi-blind channel estimator takes $\left\{\boldsymbol{s}_{p 1}, \boldsymbol{s}_{p 2}, b\right\}$ to generate a channel estimate. A semi-blind channel estimator can be expressed as $\hat{h}=G_{S B}\left(s_{p}, b\right)$ 
A.1 Channel Estimation Based on Block Type Pilot Arrangement

OFDM channel estimation symbols are transmitted periodically; in which all subcarriers are used as pilots in block-type pilot based channel estimation. The estimation can be performed by using either LS or MMSE.

\section{i. $\quad$ Least Square (LS) Estimator}

Let $A$ is the diagonal matrix of pilots as $A=\operatorname{diag}\left\{A_{0}, A_{1}, \cdots, A_{N-1}\right\}, N$ is the number of pilots in one OFDM symbol, $\hat{h}$ is the impulse response of the pilots of one OFDM symbol, and $Z$ is the AWGN channel noise.

If there is no ISI, the signal received is written as [4]

$B=A F \hat{h}+Z$

where $B$ is the vector of output signal after OFDM demodulation as $B=\left[B_{0}, B_{1}, \cdots, B_{N-1}\right]^{T},{ }^{T}$ is transpose,

Also $F$ is the Fourier transfer matrix as below,

$$
F=\left[\begin{array}{lll}
W_{N}^{00} & \cdots & W_{N}^{0(N-1)} \\
\vdots & \ddots & \vdots \\
W_{N}^{(N-1) 0} & \cdots & W_{N}^{(N-1)(N-1)}
\end{array}\right]
$$

Where weights of Fourier matrix $W_{N}^{i, k}=\frac{1}{\sqrt{N}} e^{-j 2 \pi\left(\frac{i k}{N}\right)}$

The cost function of LS algorithm is written as,

$$
\begin{aligned}
K & =|B-A F \hat{h}|^{2} \\
& =(B-A F \hat{h})^{H}(B-A F \hat{h}) \\
& =B^{H} B-B^{H} A F \hat{h}-A^{H} B F^{H} \hat{h}^{H}+A^{H} F^{H} \hat{h}^{H} A F \hat{h}
\end{aligned}
$$

Where ${ }^{H}$ denotes conjugate transpose.

The purpose of LS algorithm is to minimize the cost function $K$ without noise. For the minimization of $K$, let $\frac{\partial K}{\partial \hat{h}^{H}}=0$.

Then, from Equation

$$
\begin{aligned}
\frac{\partial K}{\partial \hat{h}^{H}} & =0-0-\frac{\partial K}{\partial \hat{h}^{H}}\left(A^{H} B F^{H} \hat{h}^{H}\right)+\frac{\partial K}{\partial \hat{h}^{H}}\left(A^{H} F^{H} \hat{h}^{H} A F \hat{h}\right) \\
& =-F^{H} A^{H} B+F^{H} A^{H} A F \hat{h} \\
& =0
\end{aligned}
$$

Then we could get

$$
\hat{h}_{L S}=\left(F^{H} A^{H} A F\right)^{-1} F^{H} A^{H} B=F^{-1} A^{-1} B
$$

Because $\hat{H}=F \hat{h}$, where $\hat{H}$ is the impulse response of the channel,

$$
\hat{H}_{L S}=A^{-1} B
$$

ii. Minimum Mean Square Error (MMSE)

\section{Estimator}

Let us denote the error of channel estimatione as

$e=H-\hat{H}$

where $H$ is actual channel estimation and $\hat{H}$ is raw channel estimation, respectively.

And the MSE of channel estimation is [5]

$$
\begin{aligned}
E\left\{|e|^{2}\right\} & =E\left\{|H-\hat{H}|^{2}\right\} \\
& =E\left\{(H-\hat{H})(H-\hat{H})^{H}\right\}
\end{aligned}
$$

where $\mathrm{E}\{\}$ is the expectation.

Let us denote the auto-covariance matrixes of $H, B$ by $S_{H H}, S_{B B}$ respectively, and cross covariance matrix between $H$ and $B$ by $S_{H B}$. Let $\sigma_{Z}^{2}$ is the noise-variance, since the channel and AWGN are not correlated, MMSE estimate of $\mathrm{H}$ is given by[6]

$\hat{H}_{M M S E}=S_{H B} S_{B B}^{-1} B$

Where

If $S_{H H}$ and $\sigma_{Z}^{2}$ are known to the receiver, CIR could be calculated by MMSE estimator as below

$\hat{H}_{M M S E}=S_{H B} S_{B B}^{-1} B$

$$
\begin{aligned}
& =S_{H H} A^{H}\left(A S_{H H} A^{H}+\sigma_{Z}^{2} I_{N}\right)^{-1} A \hat{H}_{L S} \\
& =S_{H H}\left(S_{H H}+\sigma_{Z}^{2}\left(A^{H} A\right)^{-1}\right)^{-1} \hat{H}_{L S}
\end{aligned}
$$

\section{A.2 Channel Estimation Based on Comb-Type Pilot Arrangement}

For comb-type pilot based channel estimation, the $N_{p}$ pilot signals are uniformly

Inserted into $A(k)$ according to the following equation [7]

$$
\begin{aligned}
A(k)= & A(l M+m) \\
& = \begin{cases}A_{p}(k) & m=0,1, \ldots ., M-1 \\
\text { inf.Data } & m=1,2, \ldots \ldots, M-1\end{cases}
\end{aligned}
$$

where $\mathrm{M}=$ No. of subcarriers $(N) /$ No. of pilot $\left(N_{p}\right)$

$l=$ pilot carrier index

\section{i. Piecewise Constant Interpolation}

Channel is estimated by previous pilot in Piecewise constant interpolation. And the channel estimation is given by,

$\hat{H}(k)=\hat{H}(l M+m)=\hat{H}_{p}(l), \quad 0 \leq m \leq M, \quad l=0,1, \cdots, N_{p}-1$ where $\mathrm{M}=$ No. of subcarriers $(N) /$ No. of pilot $\left(N_{p}\right)$ $l=$ pilot carrier index

\section{ii. Linear Interpolation}

Linear interpolation performs better than the piecewise constant interpolation [9]. The channel estimation at the data-carrier $k$,

$l M<k<(l+1) M$, using linear interpolation is given by:

$$
\begin{aligned}
& H(k)=H(l M+m) \quad 0 \leq m<M \quad l=0,1, \cdots, N_{p}-1 \\
& =\left(H_{p}(l+1)-H_{p}(l)\right) \frac{m}{M}+H_{p}(l) .
\end{aligned}
$$

\section{iii. Second Order Interpolation}


The channel estimation at the data subcarrier is calculated Meng-Han Hsieh and Che-Ho We [13] discussed the by used linear combination of three adjacent pilots in channel estimation methods based on comb-type pilot subSecond order interpolation technique and this technique is carrier arrangement for OFDM systems. For comb type better than linear interpolation [10]. Fig.8 shows the pilot arrangement channel estimation first the channel diagram of second order interpolation. As high order estimation algorithms are used to estimate the channel at interpolation yields better channel estimation because of pilot subcarriers and then interpolation techniques are used using more pilots and the channel estimation will be close to estimate the channel at data subcarriers. LSE algorithm to the true channel response. But computation complexity is simple. The MMSE estimator performs much better than also is increased as increasing of order.

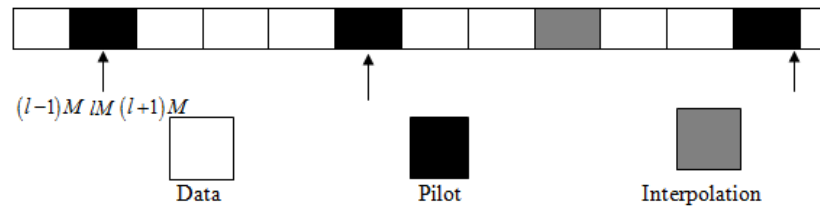

Fig. 8 Sketch map of second order interpolation [10] LSE estimator but, because of the required matrix inversions, the computation is very complex when the number of subcarriers of OFDM system increases.

A new approach to low-complexity channel estimation in OFDM systems has been presented by Ove Edfors, Magnus Sandell, Jan-Jaap van de Beek, Sarah Kate Wilson and Per Ola BAorjesson [15]. A low rank approximation is applied to a LMMSE estimator that uses the frequency correlation of the channel. SVD can also be used to derive an optimal low-rank estimator.

The channel estimation of second order interpolation is In Kala Praveen Bagadi and Prof. Susmita Das [16] a given by

$$
\begin{gathered}
\hat{H}(k)=\hat{H}(l M+m)=d_{1} \hat{H}_{p}(l-1)+d_{0} \hat{H}_{p}(l)+d_{-1} \hat{H}_{p}(l+1) \\
l=0,1, \cdots, N_{p}-1 \quad 0 \leq m \leq M,
\end{gathered}
$$
comparison has been made for channel estimation based

where $\mathrm{M}=$ No. of subcarriers $(N) /$ No. of pilot $\left(N_{p}\right)$ $l=$ pilot carrier index.

\section{ii. Cubic Spline Interpolation}

The cubic spline interpolation is given by [11]

$$
\begin{aligned}
\hat{H}(k)= & \hat{H}(l M+m) \\
= & c_{1} \hat{H}_{p}(l+1)+c_{0} \hat{H}_{p}(l)+M c_{1} \hat{H}_{p}^{\prime}(l+1)-M c_{0} \hat{H}_{p}^{\prime}(l) \\
& l=0,1, \cdots, N_{p}-1 \quad 0 \leq m \leq M,
\end{aligned}
$$

on both block-type pilot and comb-type arrangements in both SISO and MIMO OFDM based systems. In comb type pilot arrangement channel estimation is achieved by applying the channel estimation algorithms at the pilot frequencies and the interpolation of the channel at data frequencies. The MMSE estimator assumes a priori knowledge noise variance and channel covariance.

A new pilot aided channel estimation algorithm for MIMO-OFDM system over frequency selective channel has been proposed by F.Delestre and Y.Sun [17]. To estimate the channel, pilots are first transmitted in this channel estimation algorithm. In order to decode the data in each block of OFDM system, pilots are sent at the beginning of each OFDM block. The proposed channel estimation method for different numbers of transmits and receives antennas and concluded that the proposed scheme has only $4 \mathrm{~dB}$ loss when compared with ideal case. estimated channel $\left\{H_{p}(k), k=0,1, \cdots, N_{p}-1\right\}$, first

convert it to

For high rate transmissions over wireless frequencytime domain by IDFT. Then, by using the basic multirate signal processing properties [25], the signal is interpolated by transforming the $N_{p}$ points into $N$ points.

The estimate of the channel at all frequencies is obtained by:

$$
H(k)=\sum_{n=0}^{N-1} J_{N}(n) e^{-j(2 \pi / N) n k}, \quad 0 \leq k \leq N-1
$$

\section{LITERATURE REVIEW}

selective fading channels Xiaodong Cai and Georgios B. Giannakis [18]give a promising pilot symbol assisted channel estimation technique. Considering LSE and MMSE channel estimators, they have analyzed the symbol error rate (SER) performance of OFDM with M-ary phaseshift keying (M-PSK) modulation over Rayleigh-fading channels, in the presence of channel estimation errors.

To minimize the SER by minimizing the performance loss due to channel estimation errors, they also optimized the number of pilot symbols, placement of pilot symbols, and An extensive literature has been reviewed related to the power allocation between pilot and information channel estimation of communication channel. Some of symbols. the relevant work has been discussed as: 
Least Mean Square (LMS), Normalized LMS (NLMS) and 5. Recursive Least Square (RLS) algorithm three techniques to estimate the channel responses are proposed by $\mathrm{K}$. Elangovan and Dr. PLK Priyadarsini [19]. These three equalization techniques are used to cancel the fading effect due to multi-path delay spread. NLMS shows the better performance compared to LMS to reduce the bit error rate (BER). An adaptive equalization algorithm known as RLS can be adopted to further improve the performance of OFDM system.

Zhang Jie [20], DFT-based channel estimation method uses the symmetric property and calculates the changing rate of the leakage energy in order to select useful paths. The improved method can reduce the leakage energy efficiently. The MSE and BER performance of the improved method are both better than LS estimation and conventional DFT-based channel estimation method. The improved method achieves a satisfying tradeoff between complexity and performance.

\section{CONCLUSION}

A review of different channel estimation techniques has been discussed. Different channel estimators such as pilot based estimator, blind channel estimator and semi-blind channel estimators have been discussed and it is concluded that pilot based channel estimation is far better than others, because blind and semi-blind channel estimator use mathematical information about the transmitted data and become complex. Block type arrangement and comb type arrangement for pilot insertion have been reviewed and compared. Block type arrangement is used for slow fading channel whereas comb type arrangement is used for fast fading channel. In block type arrangement includes algorithms like LSE, MMSE whereas comb type arrangement includes interpolation techniques such as piecewise constant interpolation, linear

interpolation, second order interpolation, cubic spline interpolation and time domain interpolation techniques. It has been found that the performance of MMSE is much better than LSE but computation is very complex when number of subcarrier of OFDM increases. However, applying the DFT on the estimated output of these algorithms the results can be improved. DFT based channel estimation technique allows the reduction of noise component owing to operation in the transform domain and thus providing higher estimation accuracy.

\section{REFERENCES}

1. Siavash M. Alamouti, "A Simple Transmit diversity Technique for Wireless Communications", IEEE Journal on Select Areas in Communications, Vol. 16, No. 8, October 1998.

2. PAN Pei-sheng, ZHENG Bao-yu, "Channel estimation in space and frequency domain for MIMO-OFDM systems", ELSEVIER journal of China Universities of Posts and Telecommunications, Vol. 16, No. 3, pp.40-44, June 2009

3. Lavish Kansal et al., "Performance Analysis of MIMO-OFDM System Using QOSTBC Code Structure for M-QAM", Canadian Journal on Signal Processing, 2(2), May 2011

4. .Meng-Han Hsieh, Che-Ho Wei, "Channel Estimation For OFDM Systems Based On Comb-Type Pilot Arrangement In Frequency Selective Fading Channels", IEEE Transactions on Consumer Electronics, Vol. 44 , No. 1, pp. 217-225, Feb 1998.
Van de Beek, J.-J. Edfors, O. Sandell, M. Wilson, S.K. Borjesson, "On Channel Estimation in OFDM Systems", Vehicular Technology Conference, 1995 IEEE 45th, Vol. 2, pp. 815-819, July 1995.

6. M. R. McKay and I. B. Collings, "Capacity and performance of MIMO-BICM with zero-forcing receivers", IEEE Trans. Commun., vol. 53, no. 1, pp. 74-83, Jan. 2005.

7. Sarada Prasanna Dash , "Channel Estimation in Multicarrier Communication Systems", Thesis, National Institute of Technology Rourkela, 2005-09.

8. L. J. Cimini, "Analysis and simulation of a digital mobile channel using orthogonal frequency division multiplexing,', IEEE Trans. Commun., vol. 33, no. 7, pp. 665-675, Jul. 1985.

9. K.C., "An Introduction to Statistics", Vol. 2, Lecture Notes, IMM, DTU, 2005.

10. Y. Shen, Ed Martinez, "Channel Estimation in OFDM Systems", Freescale Semiconductor, pp.1-9, 2006.

11. Y. Zhao and A. Huang, "A novel channel estimation method for OFDM Mobile Communications Systems based on pilot signals and transform domain processing", in Proc. IEEE 47th Vehicular Technology Conf., Phoenix, USA, pp.2089-2093, May 1997.

12. A. V. Oppenheim and R. W. Schafer, "Discrete-Time Signal Processing", New Jersey: Prentice-Hall Inc., 1999.

13. 13.Meng-Han Hsieh and Che-Ho We "Channel Estimation for OFDM Systems based on Comb-Type Pilot arrangement in frequency selective fading channels", IEEE Transaction on Wireless Communication,vol.2, no. 1, pp 217-225, May 2009.

14. S.Galih, R.Karlina, A.Irawan, T.Adiono, A.Kurniawan, Iskandar, "Low Complexity Partial Sampled MMSE Channel Estimation for Downlink OFDMA IEEE 802.16e System', Intelligent Signal Processing and Communication Systems, 2009. ISPACS 2009. International Symposium, pp. 162-166, Jan 2009.

15. Ove Edfors, Magnus Sandell, Jan-Jaap van de Beek, Sarah Kate Wilson and Per Ola BAorjesson, "OFDM Channel Estimation by Singular Value Decomposition", IEEE Transactions on Communications, vol. 46, no.3, pp. 931-939, July 1998.

16. Kala Praveen Bagadi \& Prof. Susmita Das, "MIMO-OFDM Channel Estimation Using Pilot Carries", International Journal of Computer Applications (0975-8887) vol. 2, no.3, pp. 81-88, May 2010.

17. F.Delestre and Y.Sun, "Pilot Aided Channel Estimation for MIMOOFDM Systems", Proceedings of London Communications Symposium, pp 356-360, June 2009.

18. Xiaodong Cai and Georgios B. Giannakis "Error Probability Minimizing Pilots for OFDM With M-PSK Modulation Over Rayleigh-Fading Channels", IEEE Transactions on Vehicular Technology, vol. 53, no. 1, pp. 146-153, January 2004.

19. K. Elangovan and Dr. PLK Priyadarsini, "Performance Enhancement Technique For OFDM Using Channel Estimation Technique", International Journal of Engineering Science and Technology, vol. 2,no. 8, pp. 3483-3493, May 2010.

20.Zhang Jie, Huang Liqun, "An Improved DFT-based Channel Estimation Algorithm for MIMO-OFDM Systems', IEEE, vol.2, pp.3929-3932, 2011. 